\title{
An improved AAV vector for neurological correction of the mouse model of Mucopolysaccharidosis IIIA
}

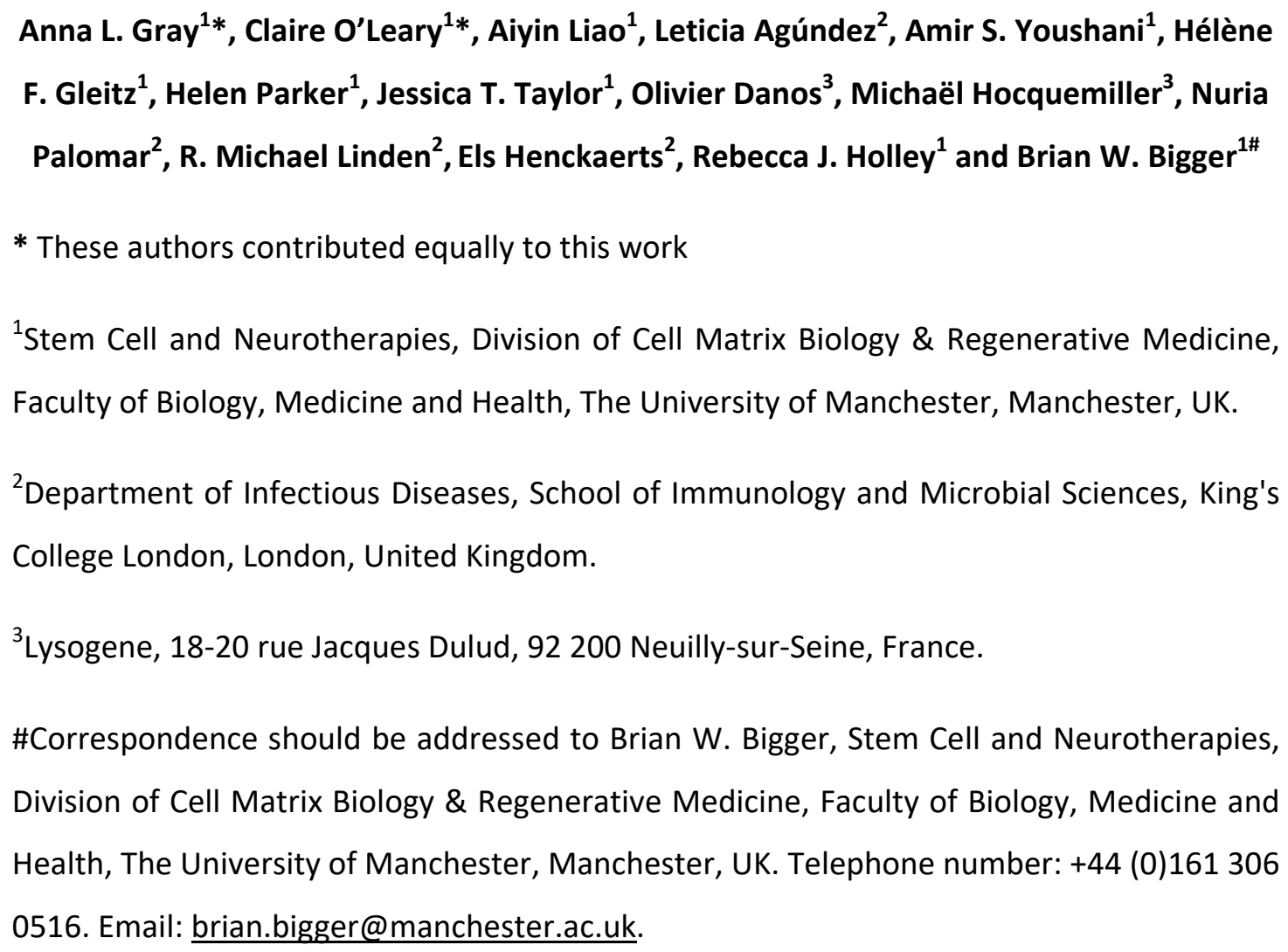

Key words: AAV, Mucopolysaccharidoses, gene therapy, lysosomal storage disease

Short title: AAV-gene therapy for MPSIIIA 


\begin{abstract}
Patients with the lysosomal storage disease mucopolysaccharidosis IIIA (MPSIIIA) lack the lysosomal enzyme $\mathrm{N}$-sulfoglucosamine sulfohydrolase (SGSH), one of the many enzymes involved in degradation of heparan sulphate. Build-up of undegraded heparan sulphate results in severe progressive neurodegeneration for which there is currently no treatment. Experimental gene therapies based on gene addition are currently being explored. Following pre-clinical evaluation in MPSIIIA mice, an AAVrh10 vector designed to deliver SGSH and sulfatase modifying factor 1 (SAF301) was trialled in four MPSIIIA patients showing good tolerance and absence of adverse events with some improvements in neurocognitive measures. Here we aimed to further improve SAF301 by removing sulfatase modifying factor 1 (SUMF1) and assess if expression of this gene is needed to increase the SGSH enzyme activity (SAF301b). Secondly, we exchanged the murine phosphoglycerate kinase (PGK) promotor with a chicken beta actin/CMV composite (CAG) promotor (SAF302) to see if we could further boost SGSH expression levels. The three different vectors were administered to MPSIIIA mice via intracranial injection and SGSH expression levels were compared 4-weeks post-treatment. Removal of SUMF1 resulted in marginal reductions in enzyme activity. However, promotor exchange significantly increased the amount of SGSH expressed in the brain leading to superior therapeutic correction with SAF302. Biodistribution of SAF302 was further assessed using GFP (SAF302GFP), indicating that vector spread was limited to the area around the injection tract. Further modification of the injection strategy to a single depth with higher injection volume increased vector distribution leading to more widespread GFP distribution and sustained expression, suggesting this approach should be adopted in future trials.
\end{abstract}




\section{Introduction}

Mucopolysaccharidosis type III (MPSIII) is a lysosomal storage disorder caused by a deficiency in enzymes that degrade heparan sulphate (HS). Loss of the lysosomal enzyme $\mathrm{N}$-sulfoglucosamine sulfohydrolase $(\mathrm{SGSH})^{1}$ causes MPSIIIA, the most common MPSIII subtype ${ }^{2}$. SGSH deficiency leads to a cascade of pathological events resulting in significant neurological manifestations with a median lifespan of 15 years $^{2}$. Patients have mild visceral symptoms, however the CNS is severely affected with neuropathology characterised by accumulation of HS, gangliosides, cholesterol and neuroinflammation ${ }^{3}$. Therefore, the focus of new therapies is to treat the neurological manifestations associated with this disease ${ }^{4}$. Replacement enzyme approaches are used to treat somatic disease in other MPS subtypes, however due to the inability of the enzyme to cross the blood brain barrier, this approach is ineffective for the treatment of MPSIIIA. To overcome this, intra-cerebrospinal fluid injection of recombinant human heparan $\mathrm{N}$-sulfatase has been tested in both mice and $\operatorname{dogs}^{5,}{ }^{6}$; this treatment provides a reduction in HS levels, which results in improvements in neuropathology and associated symptoms. Nonetheless, repeat enzyme injections generated a humoral response. Recent clinical trials of intrathecal delivery of exogenous enzyme (NCT01155778 and NCT02350816), delivered via lumbar ports to direct enzyme towards the brain, was beset with device failure issues and was ultimately unsuccessful, despite CSF HS reductions ${ }^{7}$.

Allogeneic haematopoietic stem cell transplantation (HSCT) is currently used effectively to treat neurological disease in a handful of lysosomal disorders ${ }^{8-10}$, including related MPSI, a disorder whose primary storage substrate is HS, similar to MPSIIIA. Although the success of HSCT stems from the migration of myeloid cells across the blood brain barrier and cross correction of neighbouring cells, HSCT has been shown to be ineffectual in treating MPSIIIA $^{11-13}$. Mouse models have shown this is likely due to inadequate enzyme levels achieved in the brain of MPSIII mice ${ }^{14,15}$. Myeloid-specific haematopoietic stem cell gene therapy (HSCGT), relying on gene addition in patients' own haematopoietic cells has been shown to correct neurological manifestations in a murine model of MPSIIIA ${ }^{16}$. This study showed that elevating expression levels in the brain above a threshold of $8.5 \%$ of normal SGSH was sufficient to improve primary storage, neuropathology and behaviour ${ }^{16,17}$. 
Another approach to treat lysosomal diseases is the use of adeno-associated virus (AAV) gene therapy vectors to deliver the therapeutic transgene ${ }^{18}$. A number of serotypes isolated from human and non-human primates have shown to efficiently transduce the CNS, most prominently AAV9 and AAVrh10 ${ }^{19,20}$. AAV9 can cross the blood-brain barrier and reach the CNS after intravenous administration; the quantity and manufacturing cost of vector required to reach the brain to achieve clinical efficacy in humans is extremely high and carries an elevated risk of inducing immune responses and systemic toxicity ${ }^{21}$. Therefore, a direct delivery approach into the brain parenchyma may be favourable.

Direct delivery of AAV has been used to deliver therapeutic transgenes in a number of preclinical and clinical studies of neurological lysosomal disorders ${ }^{22-26}$. In a recent study, five-week old MPSIIIA mice received a unilateral intracranial injection of AAVrh10, combining SGSH and SUMF1 in a single vector ${ }^{26}$. The inclusion of sulfatase modifying factor 1 (SUMF1) was based on the observation that co-expression of this factor enhances enzyme expression in a number of sulfatase deficiencies ${ }^{25,27,28}$. Dual gene delivery resulted in an ipsilateral restoration of SGSH and reduction in HS storage, and consequently a decrease in activated microglia. At later timepoints after treatment, a decline in GM3 ganglioside accumulation and ubiquitin-positive lesion formation was also observed. The same vector, SAF301, was later used in a Phase I/II clinical trial for MPSIIIA $^{23}$, in which patients received $7.2 \times 10^{11}$ viral genomes simultaneously via 6 injection sites at two depths in $60 \mu \mathrm{L}$ deposits bilaterally to the white matter anterior, medial and posterior to the basal ganglia. They reported no signs of toxicity; the treatment was well tolerated and clinical assessment suggested possible improvements in neuropsychological and behavioural measures. Nonetheless, although these data are encouraging they do suggest that this vector could be improved to increase therapeutic effect. This can potentially be achieved by codon optimising the transgene, using a more robust promotor to drive gene expression and/or improving the viral capsid.

In this study we compared the existing AAV vector used in the above clinical study, AAVrh10:PGK-SGSH-IRES-SUMF1 (SAF301), against two new variants, AAVrh10:PGK-SGSH (SAF301b) generated by the removal of SUMF1, and AAVrh10:CAG-SGSH (SAF302), generated by the exchange of the murine phosphoglycerate kinase (PGK) promoter with 
the chicken beta actin/CMV composite promoter in SAF301b. Expression from these vectors was assessed 4-weeks after intracranial injection into MPSIIIA mice. Vector distribution of the best vector, SAF302 carrying a GFP payload, was assessed via two-depth intrastriatal or single depth intra-striatal injections. We report here that removal of SUMF1 from SAF301 slightly reduces enzyme activity while CAG-driven SGSH expression leads to a more effective treatment of MPSIIIA mice. We could show that SAF302 was effectively distributed through the front and mid part of the brain, and was more effectively distributed by single injections rather than two-depth injections, where vector spread was more limited to the tissue immediately surrounding the injection track. These data suggest that the SAF302 variant should be used in future clinical trials.

\section{Materials and Methods}

Mice

Mice were housed in groups of two to five per cage and maintained at $21 \pm 1{ }^{\circ} \mathrm{C}$, with a constant humidity of $45-65 \%$, on a 12 -h light dark cycle [08.00 on: 20.00 off] with ad libitum access to food and water. These studies were approved by the Ethics Committee of the University of Manchester. They were conducted under licence from the Home Office in accordance with UK legislation and the European Communities Council Directive 86/609/EEC for the care and use of experimental animals. MPSIIIA mice ${ }^{29}$ on a C57BL/6J background (B6.Cg-Sgsh ${ }^{\text {mps3a } / 6 \mathrm{~J})}$ were maintained by heterozygote breeding and genotyped as previously described ${ }^{3,15,30}$.

\section{AAV production and titration}

The SAF301b transgene plasmid (ITR-PGK-SGSH-ITR) was constructed by digesting SAF301 (ITR-PGK-SGSH-IRES-SUMF-ITR) with Sacl to remove the SUMF1 gene. This digestion gave rise to a deletion of $200 \mathrm{bp}$ of the SGSH gene. This missing part was replaced by inserting a PCR fragment generated with oligos $F$ (5'-CCAGAGCTCTACGACCGGAGCCGGGA) and R (5'CCAGAGCTCTCACAGCTCATTGTGGAGG) on SAF301. This fragment was purified and digested with Sacl. Insert and vector were ligated overnight, microdialized and electroporated into $\mathrm{DH} 5 \alpha$ competent cells. Positive colonies were further analysed by sequencing. 
The SAF302 transgene plasmid (ITR-CAG-SGSH-ITR) was constructed by replacing the GFP coding sequence in the pTRUF-11 plasmid (ATCC, MBA-331) with SGSH cDNA into the NotI and Sphl sites. The original pTRUF-11 plasmid was used to generate SAF302GFP. Recombinant AAVrh10 vectors were produced, purified and titered as previously described $^{31}$.

Intracranial Injections

8-14 week old MPSIIIA mice ( $n=5-6$ per group) received bilateral injections via two different strategies into the striatum. In the short-term arm of the study, injection volumes were $4 \mu \mathrm{L}$ per animal, with a bilateral injection of $1 \mu \mathrm{L}$ delivered at 2 depths using a Hamilton syringe. The co-ordinates used were $2 \mathrm{~mm}$ lateral to Bregma at two depths of $3 \mathrm{~mm}$ and $2 \mathrm{~mm}$. All mice received a total amount of $4.1 \times 10^{9}$ genome particles in $4 \mu \mathrm{L}$ for the delivery of SAF301, SAF301b, SAF302 or SAF302GFP and sacrificed 4-weeks post injection. In the long term arm of the study, injection volumes were $6 \mu \mathrm{L}$ per animal, with a bilateral injection of $3 \mu \mathrm{L}$ delivered at a single depth using a Hamilton syringe. All mice received a total amount of $6 \times 10^{9}$ genome particles in $6 \mu \mathrm{L}$ of SAF302GFP and sacrificed 4-months post-injection. The co-ordinates used were $2 \mathrm{~mm}$ lateral to Bregma to a depth of $3 \mathrm{~mm}$. For SAF302GFP distribution studies, 5 mice were injected per group.

\section{Sample processing}

For the determination of SGSH activity, the measurement of HS levels and the colorimetric bead arrays, samples were homogenized and sonicated in homogenization buffer ( $0.5 \mathrm{~mol} / \mathrm{L} \mathrm{NaCl}, 0.02 \mathrm{~mol} / \mathrm{L}$ Tris $\mathrm{pH} 7-7.5)$, then centrifuged at 2,200g for 15 minutes at 4 ${ }^{\circ} \mathrm{C}$, and the supernatant was collected. Protein concentration was determined using the Pierce BCA assay kit (Fisher Scientific, Loughborough, UK). For immunohistochemistry, brains were fixed in $4 \%$ paraformaldehyde for 24 hours, and then incubated in $30 \%$ sucrose $2 \mathrm{mmol} / \mathrm{L} \mathrm{MgCl}_{2} / \mathrm{PBS}$ for 48 hours before freezing at $-80^{\circ} \mathrm{C}$.

\section{SGSH assay}

SGSH activity was measured in individual hemicoronal fifths using the SGSH activity assay using 4-methylumbelliferyl- $\beta$-D-N-glucosaminide (4/MU) as previously described ${ }^{15,16}$. 
Heparan sulphate analysis

Glycosaminoglycan chains were purified, AMAC labelled and analysed by RP-HPLC as previously described ${ }^{14,32,33}$. Brain extract from each specific brain region representing regions 1 to 5 from 2-3 mice within each experimental group was pooled for analysis of total levels of HS. The two pools per group (one pool for SAF301 due to technical issues) were then analysed independently for each region running each pool twice over the HPLC. Results are presented as an average of these two biological pools. Only one pool was used for region 5 due to limiting sample.

Detection of anti-AAV antibodies

Total antibody responses against AAV capsid proteins were measured in brain homogenates with an enzyme linked immunosorbent assay (ELISA) as previously described $^{31}$. Serum samples from naïve mice injected with an adjuvant plus the specific AAV vector used for each treatment group (SAF301, SAF301b or SAF302) were used as a positive control. Antibody levels were measured across two-fold serial dilutions of either the serum from positive control samples or brain homogenate collected from all experimental samples. All brain regions from each mouse within each group were pooled in appropriate volumes to generate a sample representing whole brain for each mouse.

\section{Evaluation of cytokine levels}

The levels of IL-1 $\alpha$, MCP-1, MIP-1 $\alpha$, RANTES, G-CSF, GM-CSF and KC were measured using the BD Cytometric Bead Array (CBA) Flex Set kits (BD Biosciences, Oxford, UK) as previously described ${ }^{3,34}$. Pooled samples from the right and left hemisphere of each specific brain region for each mouse were used. Five mice were analysed per experimental group for each region of the brain (1-5).

\section{Vector Copy Number}

Analysis of vector biodistribution was performed by quantitive PCR (qPCR) ${ }^{31}$. Genomic DNA from tissue homogenate was extracted using Qiagen DNeasy Blood and Tissue kit. A standard curve was generated based on specific amounts of a linearised SGSH plasmid containing native human SGSH cDNA and compared against GAPDH using naïve genomic 
murine DNA. The primer sequences used were SGSH F (5'- CCAGCATCAGAATGGGATGTA3'), SGSH R (3'-GGCGACGTAGAGGAAGAAAG-5'), GAPDH F (5'-TGCACCACCAACTGCTTAGC3') and GAPDH R (3'- AGGAACATCATCCCTGCATCC-5'). SYBR Green amplifications were performed using the PowerUp SYBR Green master mix (Thermo Fisher Scientific) to a final volume of $25 \mu \mathrm{L}$, including $5 \mu \mathrm{L}$ of DNA. Plasmid amounts were calculated to give the numbers of double-stranded vector genomes per diploid genomic equivalent.

\section{Immunohistochemistry}

Brain sections $(30 \mu \mathrm{m})$ were examined and appropriate slices were identified. Free floating immunofluorescent staining was performed on coronal sections taken at Bregma +1.7 , $+0.26,-1.18$ and $-2.62 \mathrm{~mm}$ according to the mouse brain atlas. Sections were blocked in $5 \%$ goat serum, $1 \%$ Triton-X-100 in TBS for one hour at room temperature. Sections were then incubated overnight in primary antibodies made up in blocking solution, at $4^{\circ} \mathrm{C}$ on a rocker. Sections were washed 4 times in TBS and then incubated with secondary antibody diluted in blocking solution for one hour. Following one wash in TBS, sections were incubated in DAPI (300nM) for 15 minutes. After 4 washes, sections were mounted and cover-slipped. The primary antibodies used for the co-labelling experiments in this study were as follows: chicken anti-GFP (Abcam, Cambridge, UK), rabbit anti-NeuN (Abcam, Cambridge, UK) and rabbit anti-GFAP (DakoCytomation, Ely, UK). Secondary antibodies used were AlexaFluor 488 goat anti-chicken (Life Technologies) and AlexaFluor 594 goat anti-rabbit (Life Technologies). Images were acquired on a 3D-Histech Pannoramic-250 microscope slidescanner using a $20 \mathrm{x}$ objective (Zeiss) and the DAPI, TRITC and FITC filter sets. Snapshots of the slide-scans were taken using the Case Viewer software (3D-Histech). Quantification of GFP ( $n=5$ mice per group) was performed using ImageJ, thresholding the total GFP positive area versus total area of the brain determined from NeuN staining.

\section{Statistical analysis}

Statistical analysis was performed using Graphpad Prism software (Graphpad software Inc.). All data were analysed by analysis of variance (ANOVA) and Tukey post-hoc test for analysis. Where standard deviations were unequal, data were log transformed to achieve normal distributions. Significance was assumed at $p<0.05$. 


\section{Results}

In order to determine the brain distribution, promoter strength and therapeutic activity of the new variants of the SAF301 vectors, MPSIIIA mice received intrastriatal injections of three vector variants expressing SGSH (SAF301, SAF301b, SAF302) and one variant where the transgene was exchanged with GFP (SAF302GFP) via bilateral injections at two depths (Figure 1A). SAF301 is the original vector used in a previous MPSIIIA clinical trial ${ }^{23}$, composed of an mPGK promoter controlling the expression of SGSH and SUMF1, which are separated by an IRES sequence, leading to the production of separate proteins from the same transcript (Figure 1B). The rationale for addition of SUMF1 was to determine if increased expression of SUMF1, an enzyme responsible for activating the sulfatase activity of SGSH by altering a cysteine residue in the active site to formylglycine, would aid enzyme activity ${ }^{25}$. SAF301b is a variant of the SAF301 vector in which the SUMF1 gene has been removed; SAF302 is altered to include expression of the SGSH transgene via the CAG promoter. Additionally, we injected a variant of SAF302 expressing GFP in order to assess vector distribution and promoter strength in the brain. All vectors were pseudotyped with the AAVrh10 capsid.

\section{Distribution of SAF302GFP after intrastriatal injection at two depths}

Injected MPSIIIA animals receiving SAF302GFP via bilateral injections at two depths were sacrificed four weeks after injection and brains were harvested. Coronal and sagittal sections were stained for GFP alongside neuronal marker NeuN and nuclear marker DAPI (Figure 1C-E) to allow determination of GFP-expressing cells. Vector spread is illustrated in coronal sections 1-4 (Figure 1C); with the positioning of coronal images 1-4 shown by the annotations on the sagittal image (figure 1D). The injection site is clearly visible at Bregma on coronal section 2 (Figure 1C), with GFP positive staining from the cortex through the striatum depicting the needle track. Little GFP-positive staining was evident within the cortex, except immediately surrounding the needle insertion site. However, clear transduction of cells within the hippocampus and striatum was evident (Figure 1D), spreading both anterior and posterior from the injection site. High magnification images of these regions stained with NeuN, clearly demonstrate predominant neuronal transduction 
with this vector (Figure 1E and F), with GFP detected in the nucleus and perinuclear cytoplasm of neurons and along neuronal processes, projecting from NeuN-positive neuronal cell bodies. Co-staining of GFP and NeuN in sagittal sections across the hemisphere also allowed vector distribution to be ascertained across the sagittal plane (Supplementary Figure 1A-F). Clear vector spread in both directions away from the injection site ( $2 \mathrm{~mm}$ lateral to Bregma) both towards and away from the midline was apparent. Wild-type (WT) animals were also injected with SAF302GFP at equivalent sites; no differences in vector distribution were seen between WT and MPSIIIA animals (data not shown).

Increases in SGSH enzyme activity are dependent upon vector design and position relative to the injection site

The efficacy of the three therapeutic AAV-SGSH vectors (SAF301, SAF301b, SAF302), were compared 4-weeks after injection, by assessing SGSH enzyme activity across the brain following division of the brain into 5 distinct regions from front to back as depicted in Figure $1 \mathrm{~A}$. The 5 brain regions from the left and right hemisphere were processed independently ( $n=5$ animals per treatment, 10 independently injected hemispheres per group, Figure 2).

The injection sites lay on the border of region 2 and region 3. Injection with all three vectors resulted in increases in enzyme levels in region 2 of the brain compared to untreated. However only treatment with SAF302 resulted in significant increases in SGSH levels, reaching approximately 2 -fold the normal level measured in WT animals $(p<0.0001$; Figure 2B). Furthermore, treatment with SAF302 was significantly better than SAF301 $(p<0.01)$ and SAF301b $(p<0.001)$. Likewise, in region 3, significant improvements in enzyme activity were observed following treatment with both SAF301 and SAF302 $(p<0.0001$; Figure 2C), with SGSH levels equal to or greater than WT enzyme. Results suggested SAF301 was superior to SAF301b, with little improvement in SGSH activity noted following SAF301b injection.

In areas more distal to the injection site (brain regions 1, 4 and 5), little difference in SGSH enzyme activity levels was apparent between untreated MPSIIIA mice and all AAV treated 
MPSIIIA mice and they were all significantly different from WT $(P<0.0001)$ (Figure 2D-F, respectively). Thus, the improvement in SGSH enzyme activity in treated MPSIIIA mice varied significantly across brain regions [ $p<0.0001]$, with regions closest to the injection site showing the highest activity. When the enzyme activity across all regions of the brain as a whole was studied (Figure 2A), both SAF301 and SAF302 significantly increased SGSH levels compared to untreated MPSIIIA animals ( $p<0.01$ and $p<0.0001$ respectively), however only SAF302 recovered enzyme activity to near WT levels.

Vector copy number (VCN) was determined by qPCR on pooled regions across the hemisphere of injected animals (Figure 2G-I and Supplementary Figure 2). VCN was not significantly different in brain tissue treated with any of the AAV-SGSH vectors (Supplementary Figure 2), although it is noted that one animal within the SAF301 group had a recorded VCN of 85 , over three-times higher than other mice within this group. VCN was also plotted against enzyme activity for each mouse in SAF301, SAF301b and SAF302 treatment groups (Figure 2G-I, respectively) with comparisons suggesting an improved functional outcome per VCN following intrastriatal injection of SAF302.

Reductions in heparan sulphate accumulation in the brains of MPSIIIA mice after SAF302 treatment

The primary storage material, HS, was measured in brain extracts from regions 1-5 from five untreated MPSIIIA, WT and AAV-injected animals (SAF301, SAF301b, SAF302). Due to limits of sample size, individual regions were processed separately, with each coronal fifth of brain from 2 or 3 animals pooled together in two independent biological samples per region prior to analysis. The amount of $\mathrm{HS}(\mu \mathrm{g})$ per $\mathrm{mg}$ brain protein was then determined by reverse phase HPLC (Figure 3). Total HS for the whole brain was calculated by averaging the values obtained across brain regions for each mouse and presenting it as fold increase over WT (Figure 3A). A significant 9.2-fold increase was seen in untreated MPSIIIA mice compared to WT mice $(p<0.0001)$, due to the accumulation of undegraded HS as a direct consequences of the absence of SGSH. Little change in total HS was achieved compared to untreated MPSIIIA animals following treatment with SAF301. Overall HS levels in SAF301b and SAF302 were reduced to 6.8 and 4.7 fold above WT, respectively, indicating a clear 
trend towards decreased HS following treatment with these vectors versus MPSIIIA., However elevation of HS compared to WT remained, presumably reflecting the incomplete transduction of all cells within the brain within these regions.

When individual brain regions were studied, total HS was increased in untreated MPSIIIA mice compared to WT mice in all regions as expected (Figure 3B-F). In regions 2 and 3, close to the injection sites, clear trends towards reduction in HS levels were observed with all AAV-treatments. However, in region 2 SAF302 appeared to provide superior reductions in HS accumulation compared to the other two vectors, reducing HS by 3.4-fold over MPSIIIA. In region 3 treatment with both SAF301b and SAF302 were equivalent and provided enhanced reductions in HS compared to SAF301. There were also reductions in total HS levels in region 1, 4 and 5 with SAF302, whereas SAF301 and SAF301b had little effect. Notably, limitations from pooling of brain sections limited the ability to demonstrate significant differences in individual regions.

Reductions in neuroinflammation were most significant in MPSIIIA mice treated with SAF302

Neuroinflammation was assessed using cytometric bead array (CBA) analysis to quantify a set of inflammatory cytokines in brain extracts from regions 1 to 5 of AAV-treated MPSIIIA mice (SAF301, SAF301b, SAF302), and compared to equivalent brain regions of age matched WT and MPSIIIA untreated mice. CBA values were averaged across brain regions for each mouse to give an overall reading for each analyte. Neuroinflammation was more outspoken in MPSIIIA mice compared to WT mice, as shown by significant increases in macrophage inflammatory protein (MIP-1 $\alpha / C C L 3 ; p<0.0001)$, monocyte chemoattractant protein (MCP-1/CCL2; $\mathrm{p}<0.0001)$ and Interleukin-1 $\alpha$ (IL-1 $\alpha ; \mathrm{p}<0.05 ;$ Figure 4A-C, respectively). Total levels of RANTES (CCL5) and keratinocyte chemoattractant (KC/CXCL1) were however similar between WT and MPSIIIA mice at this early disease stage (Figure 4D$E$, respectively). An overall reduction in MIP-1 $\alpha$ was only seen following treatment with SAF302 $(p<0.01)$. MCP-1 was significantly reduced following treatment with SAF301 $(p<0.01)$, SAF301b $(p<0.05)$ and SAF302 $(p<0.01)$ compared to untreated MPSIIIA mice. Finally, IL-1 $\alpha$ was reduced following treatment with all 3 vectors, however this was only 
significant with SAF302 $(p<0.01)$. Data for each of the analytes in individual brain regions are shown in supplementary Figure 3, demonstrating the varying response to vector treatment, dependent upon position relative to the injection site, with the most significant effects noted close to the injection site in regions 2 and 3.

Absence of brain-specific antibody response to AAV-SGSH vectors in injected MPSIIIA mice

Generation of antibodies against AAV capsid proteins can significantly reduce the effectiveness of AAV-vector therapies ${ }^{35}$. Therefore, total antibody responses to AAV were analysed in the brain of untreated and SAF301, SAF301b, SAF302 treated mice. Positive control samples were generated by subcutaneously injecting naïve mice with each AAV type mixed with an adjuvant known to induce antibody responses. Serum collected from these mice was used as a positive control sample. Anti-AAV IgG antibodies were undetectable in untreated groups, however high antibody responses were generated in positive control mice treated with adjuvant plus each AAV-type. No anti-AAV antibodies were detected in mice treated with SAF301, SAF301b or SAF302 (Figure 4F-H, respectively), suggesting there is no antibody response to AAV within the brain at least.

Sustained, neuron-specific gene transduction following intrastriatal injection of SAF302GFP at a single depth

To investigate whether distribution could be enhanced using a larger injection volume at a single depth as opposed to delivery over two depths (as in Figure 1) and to evaluate longterm expression of the vector, a second GFP distribution study was conducted, assessing mice 4-months after injection. MPSIIIA mice received stereotactic injection of AAVSAF302GFP at 8-14 weeks of age via bilateral injections at a single intrastriatal depth (Figure $5 \mathrm{~A}, \mathrm{n}=5$ animals). Animals were then sacrificed at approximately 6 months of age, around 4-months post-injection. Coronal and sagittal sections were co-labelled with NeuN, GFP and DAPI to give a comprehensive overview of vector distribution throughout the brain (Figure 5B/C). Imaging of the brain sections demonstrated enhanced vector distribution in the animals which received injections at single depth compared to the animals in the short-term study receiving two-depth injections (compare Figure 5B to Figure 1C). Maximal GFP expression was present in the locality of the injection site, 
reducing in scope and intensity with increasing distance away from this region. Coronal section 2 shows GFP positive cells throughout the striatum radiating out from the injection site. GFP-positive cells were also visible along the striatum and cortex touching the white matter tract of the external capsule and within the corpus callosum. Expression was considerably greater in coronal section 3, compared to the short-term study, with more extensive spread of GFP-positive cells within the hippocampus indicating increased spread of the vector. Internal capsule staining was also apparent, with vector spread apparent in the cerebral peduncle arising from the internal capsule in coronal section 4 (Figure 5B). High magnification images of the hippocampus and the striatum denoted by the dashed boxes in the sagittal section shown in Figure 5C, confirm the neuronal specificity of the SAF302GFP vector (Figure 5D and E, respectively) with strong co-localisation of GFP with neuronal nuclear marker NeuN, with GFP extending along neuronal processes. Co-staining of GFP with astrocyte marker glial fibrillary acidic protein (GFAP) in both short-term twodepth injected and long-term animals indicated a proportion of astrocytes were also transduced alongside neurons (Figure 6). However, the proportion of astrocytes targeted was reduced compared to neurons. No cells within the cerebellum were transduced following intrastriatal injection of SAF302GFP in the long-term study, similar to results achieved in the short-term study arm.

Expression of GFP across Bregma following use of the two injection strategies was quantified as the percentage of GFP-positive cells relative to total brain area (Figure 6C-E; $\mathrm{n}=5$ animals per group). In the vicinity of the injection site (Bregma +0.26) the single injection strategy (long-term) led to increased global GFP compared to the two depth strategy (short-term; Figure 6D). Increased transduction of cells distal to the injection site was also apparent with the single bolus injection, whereas little transduction form the double height injection at distal sites was seen (Figure 6C and E).

\section{Discussion}

Mucopolysaccharide diseases, especially subtypes with substantial neurological involvement, including MPSIIIA, are ideal candidates for gene therapy. In MPSIIIA patients the brain is most affected, therefore, direct delivery to the brain using AAV may prove 
beneficial. Vector mediated delivery and cross-correction are two potential approaches to restore enzyme levels; if a number of cells can express the deficient SGSH enzyme it can be taken up by surrounding cells. As a result, for clinical efficacy a therapeutic vector that produces sufficient levels of enzyme is critical to enable cross-correction. Here we show superior SGSH expression following intracranial injection of SAF302, providing higher levels of enzyme to the brain compared to SAF301 and SAF301b. SAF301 has previously been used in a preclinical study ${ }^{26}$ and clinical trial ${ }^{23}$. Winner et $\mathrm{al}^{26}$ demonstrated that in mice receiving SAF301 via a unilateral injection, high levels of SGSH could be detected close to the injection site, with substantially less expression detected as the distance from the injection region increased. Vector-mediated delivery of SGSH improved HS catabolism, reduced microglial activation, and led to reduced GM3 ganglioside accumulation and ubiquitin-positive lesion formation with improvements noted over time ${ }^{26}$. In patients, intracerebral administration of SAF301 at six sites per hemisphere in the white matter anterior, medial and posterior to the basal ganglia, suggested a cognitive benefit in the youngest patient. However, the therapeutic effect was less clear in older patients with presumably higher disease burden. Increasing vector potency by altering vector design may increase the clinical efficacy of this vector. Our studies were designed to firstly determine the role of SUMF1 in the SAF301 vector and secondly to determine whether improving vector design by introducing a strong ubiquitous promotor would increase SGSH expression in a murine model of MPSIIIA.

For all vectors analysed, the therapeutic efficacy was clear in the brain areas proximal to the injection site. Similar findings were reported by Winner and others $(2016)^{26}$, who utilised a unilateral intrastriatal injection protocol and reported reduced primary and secondary pathology only in areas within, or directly connected to the striatum. All variants increased SGSH enzyme levels in MPSIIIA mice, but given that no differences in VCN were seen, despite equivalent titres delivered, SAF302 was clearly superior. The removal of SUMF1 from SAF301 to generate SAF301b resulted in a 50\% reduction in SGSH expression consistent with the findings by Fraldi ${ }^{25}{ }^{27}$ whereby SUMF1 activity is required for SGSH processing. Modification of the promoter via exchange of the relatively weak PGK promoter with the stronger CAG promotor containing an intron acceptor site led to 
production of a superior vector; SGSH activity levels were similar to WT levels in mice injected with SAF302, yet only reached $30 \%$ of WT levels in mice treated with SAF301. Additionally, in brain regions adjacent to the injection site, injections with SAF302 increased SGSH activity considerably above WT, whereas levels obtained with SAF301 were much below WT levels. Thus the SAF302 variant was most effective overall ${ }^{36}$. A vector containing the CAG promotor and SGSH-IRES-SUMF1 might be the ideal scenario, although co-expression of SGSH with SUMF1 in a lentiviral context has not proven beneficial in increasing SGSH activity in our hands (data not shown).

Deficiency of the SGSH enzyme causes a progressive accumulation of undegraded HS. From our previous studies using a different therapeutic strategy, lentiviral-mediated haematopoietic stem cell transplant in the MPSIIIA mouse model, led to global increases across the brain in SGSH to $7 \%$ of WT, which was necessary to reduce HS levels and to correct neuroinflammation ${ }^{16}$, with similar results obtained in $\mathrm{MPSIIIB}^{14}$ and $\mathrm{MPSII}^{37}$ mouse models. In MPSIIIC animals injection of AAV-HGSNAT reduced resulted in a $46 \%$ reduction in HS leading to associated decreases in neuroinflammation ${ }^{38}$. All studies led to improvements in disease-associated changes in behaviour, For this treatment to be effective in the clinic, SGSH levels need to be sufficiently increased to significantly lower HS storage and associated neuroinflammation. We observed a trend towards reduced HS storage and decreases in neuroinflammatory markers MIP-1 $\alpha$, MCP-1 and IL-1 $\alpha$ in each brain hemisphere injected with SAF302. Importantly, when regions 2 and 3, which lie at the border of the injection site were studied in isolation, significant decreases in HS storage were observed. Thus, we can conclude that SAF302 produces sufficient enzyme levels close to the injection site to successfully prevent HS accumulation.

Injection of animals with the SAF302GFP vector allowed us to evaluate vector distribution in the CNS of MPSIIIA mice using two different delivery methods although vector amounts and time of sacrifice are not directly comparable $\left(3 \times 10^{9}\right.$ viral genomes per injection site in the long-term study harvesting animals 4-months post-injection and $1 \times 10^{9}$ per site at two depths $\left(2 \times 10^{9}\right.$ total $)$ in the short-term study harvesting animals at 4-weeks postinjection). In the short-term two-depth injection study and single-depth injection longterm study we observed a distinct vector distribution pattern with most intense GFP+ 
staining and thus cell transduction visible within regions 2 and 3, near the injection site. Co-staining experiments highlighted neurons as the primary cell type transduced, however a proportion of astrocytes were also targeted. There was no evidence of GFP staining within the cerebellum (region 5), thereby providing an explanation for the drop off seen in enzyme and HS levels as well as neuroinflammation reduction in regions 4 and 5 . Although cells types transduced in the short-term GFP study were similar to those seen in the longterm study despite the altered injection schedule and increased vector spread it was clear that two depth injections were less efficient than a single intrastriatal injections despite the reduced volume; the vector appeared to distribute more along the injection track in the two depth injections rather than spreading laterally from the end of the injection site as per the intrastriatal injections used in the long-term SAF302GFP study. This probably reflects reflux of the vector as the needle is withdrawn, rather than creating a larger lateral pressure that delivering a single bolus of virus achieves.

12 injection sites per patient were used in the SAF301 clinical trial, targeting white matter regions of the brain leading to reported disease stabilisation ${ }^{23} .12$ similar trajectories were used together with two additional sites in the cerebellum in a recent clinical trial in MPSIIIB (16 sites per patient), leading to small improvements or a decrease in projected neurocognitive decline, suggesting that if treatment is administered early in the disease course the treatment may have some benefit for patients ${ }^{24}$. Our results indicate that one injection site per hemisphere leads to modest vector spread and suggest that the delivery technique will need to be optimized for full clinical efficacy.

In summary, our studies show that the SGSH AAV vector variant SAF302 produced a greater in vivo benefit relative to SAF301, and highlights the value of promoter choice in vector design. GFP expression was substantial regardless of whether the mice were sacrificed at 4-weeks or 4-months post injection, confirming stability of the CAG promoter over time. The SAF302 vector could be a significant improvement over SAF301 for a future clinical trial in sanfilippo disease. 


\section{Acknowledgements}

The work was funded by grants from Lysogene SAS. The Bioimaging Facility microscopes used in this study were purchased with grants from BBSRC, Wellcome and the University of Manchester Strategic Fund. Special thanks go to Roger Meadows for his help with microscopy.

\section{Author Disclosure Statement}

This study was funded by a commercial grant from Lysogene SAS, who were also involved in the study design. BB and CoL are shareholders in Phoenix Nest Inc. and have licenced a related AAV programme for MPSIIIC. BB is a scientific advisory board member and shareholder in Orchard Therapeutics Ltd and has licenced unrelated stem cell gene therapy programmes for MPSIIIA and MPSIIIB. EH is funded by a Rare Disease Consortium Award from Pfizer Inc. to develop AAV vector technology. A patent \#WO2015121501 on "Adeno-associated virus vector" has been deposited by ML and EH. ML is a consultant to various gene therapy companies. $\mathrm{ML}$ is a scientific advisory board member of Spark Therapeutics. 
References

1. Valstar MJ, Ruijter GJ, van Diggelen OP et al. Sanfilippo syndrome: A mini-review. J Inherit Metab Dis 2008.

2. Heron B, Mikaeloff $\mathrm{Y}$, Froissart $\mathrm{R}$ et al. Incidence and natural history of mucopolysaccharidosis type III in France and comparison with United Kingdom and Greece. Am J Med Genet A 2011;1:58-68.

3. Wilkinson FL, Holley RJ, Langford-Smith KJ et al. Neuropathology in mouse models of mucopolysaccharidosis type I, IIIA and IIIB. PloS one 2012;7:e35787.

4. de Ruijter J, Valstar MJ, Wijburg FA. Mucopolysaccharidosis type III (Sanfilippo Syndrome): emerging treatment strategies. Curr Pharm Biotechnol 2011;12:923-930.

5. Hemsley KM, Norman EJ, Crawley AC et al. Effect of cisternal sulfamidase delivery in MPS IIIA Huntaway dogs-A proof of principle study. Molecular genetics and metabolism 2009;98:383-392.

6. Hemsley KM, King B, Hopwood JJ. Injection of recombinant human sulfamidase into the CSF via the cerebellomedullary cistern in MPS IIIA mice. Molecular genetics and metabolism 2007;90:313-328.

7. Jones SA, Breen C, Heap $\mathrm{F}$ et al. A phase $1 / 2$ study of intrathecal heparan-Nsulfatase in patients with mucopolysaccharidosis IIIA. Molecular genetics and metabolism 2016;118:198-205.

8. Boelens J. J. WRF, Bierings M. HSCT for inborn errors of metabolism. In: Hematopoetic Stem Cell Transplantation, The EBMT Handbook. CE Apperley J, Gluckman E, Gratwhol A, Masszi T, ed. (Forum Service Editore, Genoa, Italy). 2008; pp. pp. 545-553.

9. Saif MA, Bigger BW, Brookes KE et al. Hematopoietic stem cell transplantation improves the high incidence of neutralizing allo-antibodies observed in Hurler's syndrome after pharmacological enzyme replacement therapy. Haematologica 2012;97:1320-1328.

10. Wynn RF, Wraith JE, Mercer J et al. Improved Metabolic Correction in Patients with Lysosomal Storage Disease Treated with Hematopoietic Stem Cell Transplant Compared with Enzyme Replacement Therapy. Journal of Pediatrics 2009;154:609-611. 
11. Prasad VK, Mendizabal A, Parikh SH et al. Unrelated donor umbilical cord blood transplantation for inherited metabolic disorders in 159 pediatric patients from a single center: influence of cellular composition of the graft on transplantation outcomes. Blood 2008;112:2979-2989.

12. Shapiro EG, Lockman LA, Balthazor M et al. Neuropsychological outcomes of several storage diseases with and without bone marrow transplantation. Journal of Inherited Metabolic Disease 1995;18:413-429.

13. Sivakumur $\mathrm{P}$, Wraith JE. Bone marrow transplantation in mucopolysaccharidosis type IIIA: A comparison of an early treated patient with his untreated sibling. Journal of Inherited Metabolic Disease 1999;22:849-850.

14. Holley RJ, Ellison SM, Fil D et al. Macrophage enzyme and reduced inflammation drive brain correction of mucopolysaccharidosis IIIB by stem cell gene therapy. Brain : a journal of neurology 2018;141:99-116.

15. Langford-Smith A, Wilkinson FL, Langford-Smith KJ et al. Hematopoietic stem cell and gene therapy corrects primary neuropathology and behavior in mucopolysaccharidosis IIIA mice. Mol Ther 2012;20:1610-1621.

16. Sergijenko A, Langford-Smith A, Liao AY et al. Myeloid/Microglial driven autologous hematopoietic stem cell gene therapy corrects a neuronopathic lysosomal disease. Mol Ther 2013;21:1938-1949.

17. Bigger BW, Wynn RF. Novel approaches and mechanisms in hematopoietic stem cell gene therapy. Discov Med 2014;17:207-215.

18. Hocquemiller M, Giersch L, Audrain M et al. Adeno-Associated Virus-Based Gene Therapy for CNS Diseases. Human gene therapy 2016;27:478-496.

19. Foust KD, Nurre E, Montgomery CL et al. Intravascular AAV9 preferentially targets neonatal neurons and adult astrocytes. Nat Biotech 2009;27:59-65.

20. Cearley $\mathrm{CN}$, Wolfe JH. Transduction Characteristics of Adeno-associated Virus Vectors Expressing Cap Serotypes 7, 8, 9, and Rh10 in the Mouse Brain. Molecular therapy : the journal of the American Society of Gene Therapy 2006;13:528-537.

21. Hinderer C, Katz N, Buza EL et al. Severe Toxicity in Nonhuman Primates and Piglets Following High-Dose Intravenous Administration of an Adeno-Associated Virus Vector Expressing Human SMN. Human gene therapy 2018;29:285-298. 
22. Worgall S, Sondhi D, Hackett NR et al. Treatment of late infantile neuronal ceroid lipofuscinosis by CNS administration of a serotype 2 adeno-associated virus expressing CLN2 cDNA. Human gene therapy 2008;19:463-474.

23. Tardieu $M$, Zérah $M$, Husson $B$ et al. Intracerebral administration of adenoassociated viral vector serotype rh.10 carrying human SGSH and SUMF1 cDNAs in children with mucopolysaccharidosis type IIIA disease: results of a phase I/II trial. Hum Gene Ther 2014;25:506-516.

24. Tardieu M, Zérah M, Gougeon ML et al. Intracerebral gene therapy in children with mucopolysaccharidosis type IIIB syndrome: an uncontrolled phase 1/2 clinical trial. Lancet Neurol 2017:712-720.

25. Fraldi A, Hemsley K, Crawley A et al. Functional correction of CNS lesions in an MPSIIIA mouse model by intracerebral AAV-mediated delivery of sulfamidase and SUMF1 genes. Human molecular genetics 2007;16:2693-2702.

26. Winner LK, Beard H, Hassiotis S et al. A Preclinical Study Evaluating AAVrh10-Based Gene Therapy for Sanfilippo Syndrome. Hum Gene Ther 2016;27:363-375.

27. Fraldi A, Biffi A, Lombardi A et al. SUMF1 enhances sulfatase activities in vivo in five sulfatase deficiencies. Biochem J 2007;403:305-312.

28. Cosma MP, Pepe S, Annunziata I et al. The multiple sulfatase deficiency gene encodes an essential and limiting factor for the activity of sulfatases. Cell 2003;113:445456.

29. Bhaumik M, Muller VJ, Rozaklis T et al. A mouse model for mucopolysaccharidosis type III A (Sanfilippo syndrome). Glycobiology 1999;9:1389-1396.

30. Langford-Smith A, Langford-Smith KJ, Jones SA et al. Female mucopolysaccharidosis IIIA mice exhibit hyperactivity and a reduced sense of danger in the open field test. PloS one 2011;6:e25717.

31. Tordo J, O'Leary C, Antunes A et al. A novel adeno-associated virus capsid with enhanced neurotropism corrects a lysosomal transmembrane enzyme deficiency. Brain : a journal of neurology 2018;141:2014-2031.

32. Holley RJ, Deligny A, Wei W et al. Mucopolysaccharidosis Type I, Unique Structure of Accumulated Heparan Sulfate and Increased N-Sulfotransferase Activity in Mice Lacking alpha-L-iduronidase. Journal of Biological Chemistry 2011;286:37515-37524. 
22

33. Watson HA, Holley RJ, Langford-Smith KJ et al. Heparan sulfate inhibits hematopoietic stem and progenitor cell migration and engraftment in mucopolysaccharidosis I. J Biol Chem 2014;289:36194-36203.

34. Wilkinson FL, Sergijenko A, Langford-Smith KJ et al. Busulfan conditioning enhances engraftment of hematopoietic donor-derived cells in the brain compared with irradiation. Molecular therapy : the journal of the American Society of Gene Therapy 2013;21:868-876. 35. Rapti K, Louis-Jeune V, Kohlbrenner E et al. Neutralizing antibodies against AAV serotypes 1, 2, 6, and 9 in sera of commonly used animal models. Molecular therapy : the journal of the American Society of Gene Therapy 2012;20:73-83.

36. Dirren $E$, Towne $C L$, Setola $V$ et al. Intracerebroventricular injection of adenoassociated virus 6 and 9 vectors for cell type-specific transgene expression in the spinal cord. Hum Gene Ther 2014;25:109-120.

37. Gleitz HF, Liao AY, Cook JR et al. Brain-targeted stem cell gene therapy corrects mucopolysaccharidosis type II via multiple mechanisms. EMBO Mol Med 2018.

38. Tordo J, O'Leary C, Antunes ASLM et al. A novel adeno-associated virus capsid with enhanced neurotropism corrects a lysosomal transmembrane enzyme deficiency. Brain 2018. 
Figure legends:
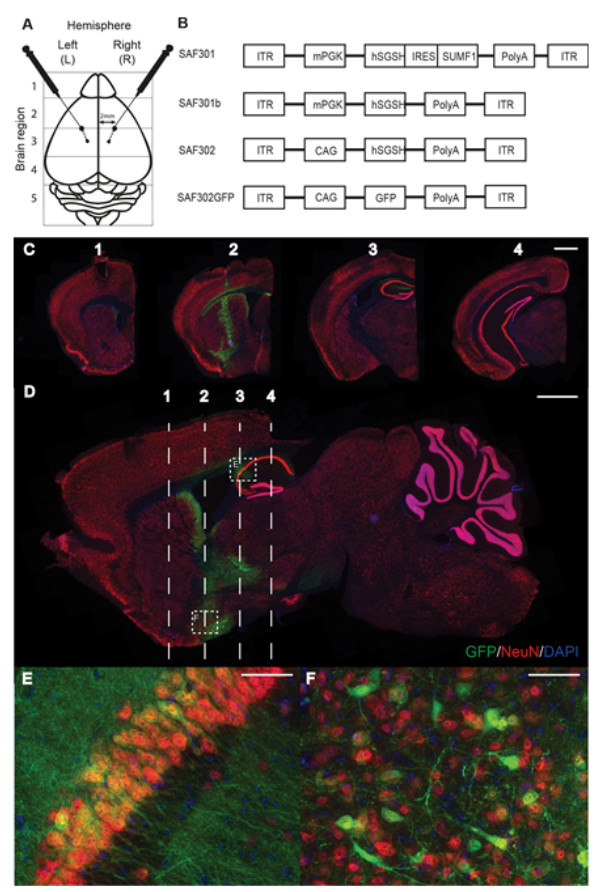

Figure 1: Intrastriatal injection of AAV vectors in MPSIIIA mice.

(A) Diagram of the intrastriatal injection site relative to the 5 different brain regions. MPSIIIA mice received stereotactic injection of AAV at 8-14 weeks of age using a Hamilton syringe ( $n=5-6$ per group). Vectors were administered at a dose of $4.1 \times 10^{9}$ genome particles in $4 \mu$ l delivered via $1 \mu \mathrm{l}$ deposits at two intrastriatal depths ( $2 \mathrm{~mm}$ and $3 \mathrm{~mm}$ ), $2 \mathrm{~mm}$ lateral to the midline in both hemispheres at the border between brain region 2 and 3.

(B) Schematic of therapeutic AAV vectors SAF301, SAF301b and SAF302, all containing the human SGSH gene and SAF302GFP.

(C-F) Mice injected with SAF302GFP were harvested 4-weeks post-surgery. Coronal brain sections from Bregma $+1.7,+0.26,-1.18$ and $-2.62 \mathrm{~mm}$ (C) and a sagittal section at $1.2 \mathrm{~mm}$ lateral to the midline (D) co-labelled with NeuN (red), GFP (green) and DAPI (blue). Scale bars $=1000 \mu \mathrm{m}$. High magnification images of the regions indicated by the dashed boxes in D show transduction of cells within the hippocampus $(E)$ and the striatum $(F)$. scale bars $=$ $50 \mu \mathrm{m}$. 

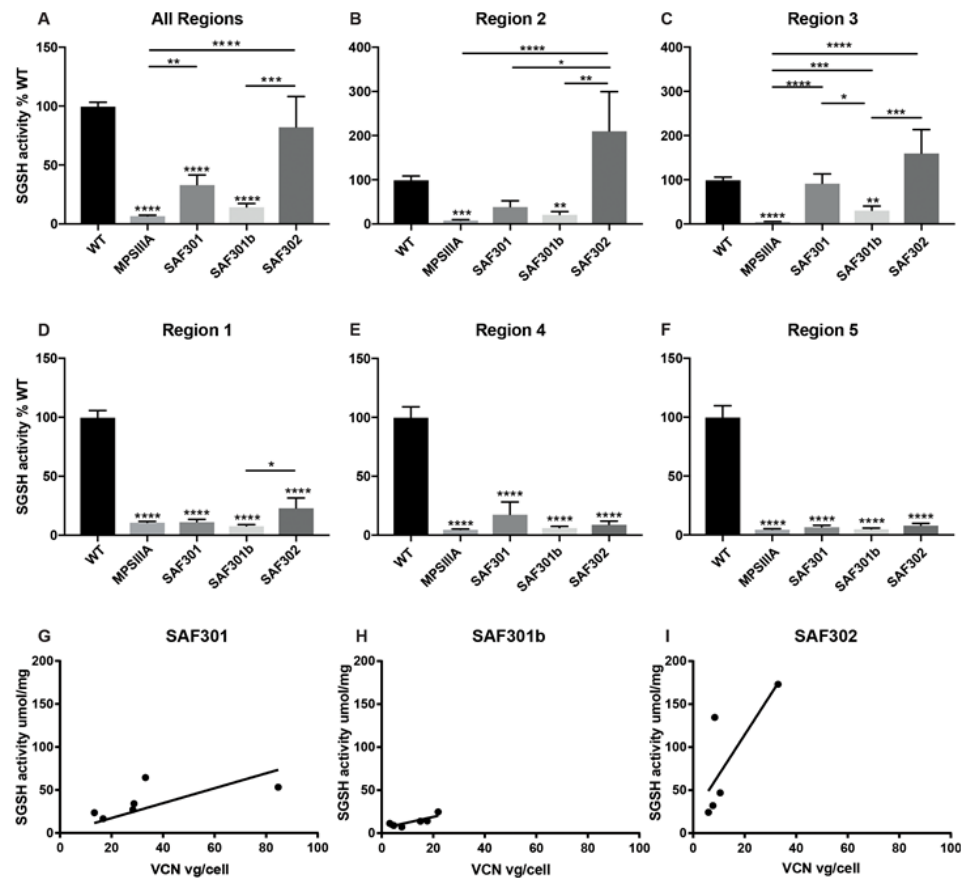

Figure 2: SGSH activity levels in AAV-treated MPSIIIA mice vary with vector type and location relative to the injection site, with the highest level of enzyme activity observed with SAF302.

(A-F) SGSH enzyme activity was measured in individual brain regions 1 to 5 according to the map given in Figure 1A. Enzyme levels from an average of all regions is given in A. SGSH enzyme levels for each individual brain region are given in region 1 (B), 2 (C), 3 (D), 4 (E) and 5 (F). Data are presented as mean percentage SGSH activity relative to WT levels \pm SEM. In regions 1-5 (B-F) SGSH levels were log transformed prior to statistical analysis. $* * * * p<0.0001, * * * p<0.001, * * p<0.01$, and $* p<0.05$ calculated from one way ANOVA with Tukey's multiple comparisons test. Significance to WT is shown above bars, all other comparisons are shown using a line.

(G-I) Vector copy number was assessed across brain regions for each animal and plotted against the enzyme activity for the SAF301 (G), SAF301b (H) and SAF302 (I) treatment groups. 

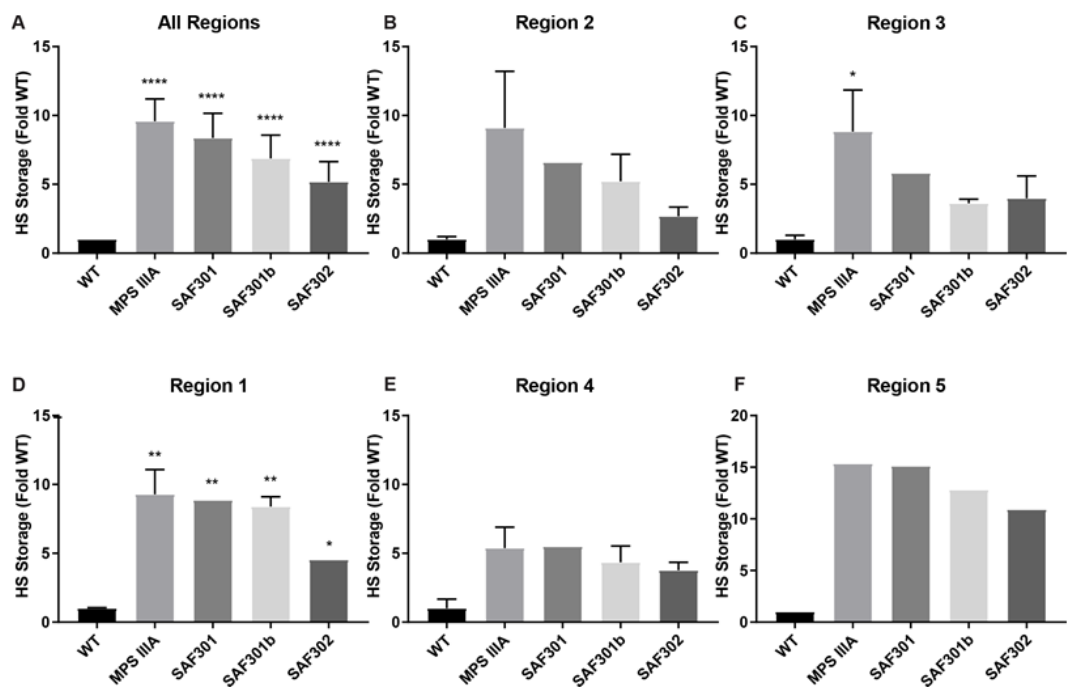

Figure 3: Total amount of HS determined by RP-HPLC analysis of AMAC-labelled disaccharides.

(A-F) HS was quantified separately in regions 1-5 and expressed as a fold-change over WT \pm SEM. Average amounts of HS across pooled brain regions (average of regions 1-5; A) and individual brain regions 1-5 (B-F) are shown. Animals were pooled prior to analysis with two or three animals per pool, with two pools per group, except SAF301 where only one pool was analysed. Error bars represent SEM of the average of each pool. Data was log transformed prior to analysis with using a one way ANOVA with Tukey's multiple comparisons test. Significant differences against WT are shown above bars $* * * * p<0.0001$, $* * p<0.01, * p<0.05$. 

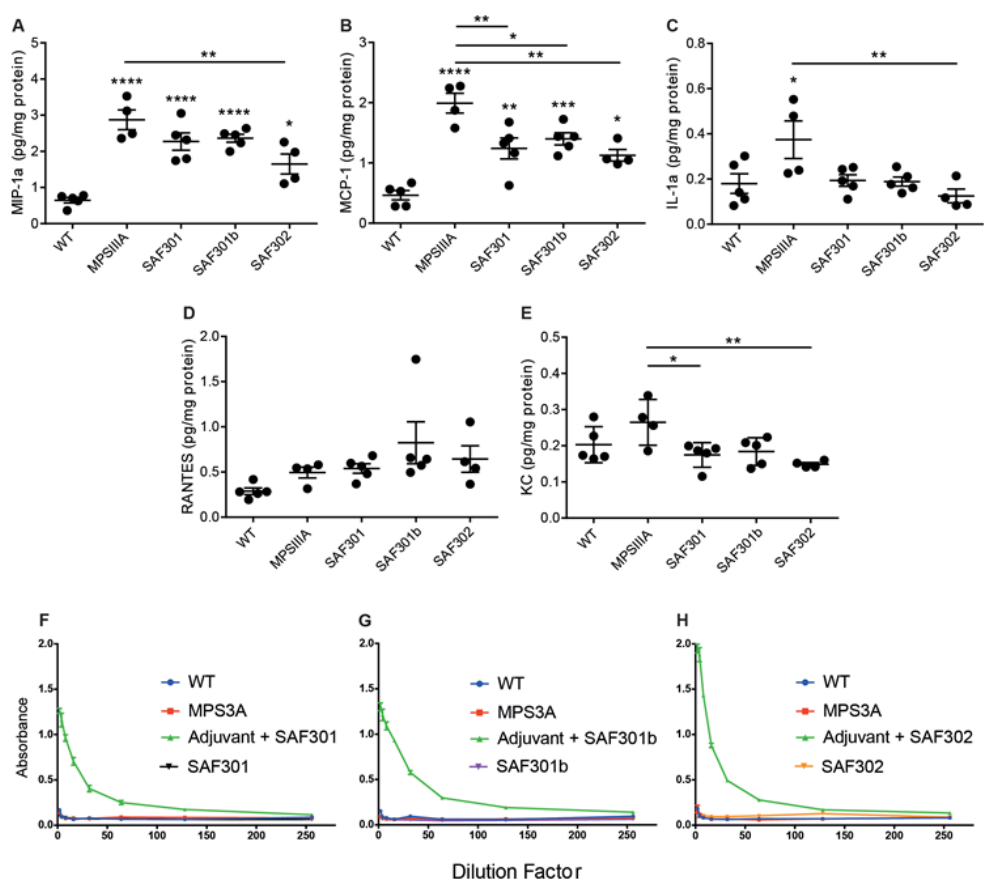

Figure 4: Assessment of neuroinflammation and AAV antibody responses in AAV- treated MPSIIIA mice.

(A-E) Protein levels for MIP-1 $\alpha$ (A), MCP-1 (B), IL-1 $\alpha$ (C), RANTES (D) and KC (E) as measured by CBA flex sets. Each data point represents the value for each individual mouse across all brain regions where a signal was measurable. For MIP-1 $\alpha$, RANTES and KC an average of brain regions 1-5 are shown, for MCP-1 an average of brain regions 2-5 are shown and for IL-1 $\alpha$ an average of brain regions $1-4$ are shown. ${ }^{*} p<0.05, * * p<0.01, * * * p$ $<0.001,{ }^{* * * *} p<0.0001$ and $p$ values are from one way ANOVA with Tukey's multiple comparisons test. Significance to WT is shown above bars, all other comparisons are shown using a line.

(F-H) Concentrations of total IgG antibody responses against AAV in the brains of WT untreated, MPSIIIA untreated and AAV-SAF301 (F), SAF301b (G) and SAF302 (H) treated MPSIIIA mice were measured using an anti-AAV antibody ELISA. The positive control consisted of serum samples collected from naïve animals, which received injections with adjuvant and the various AAV vectors. 

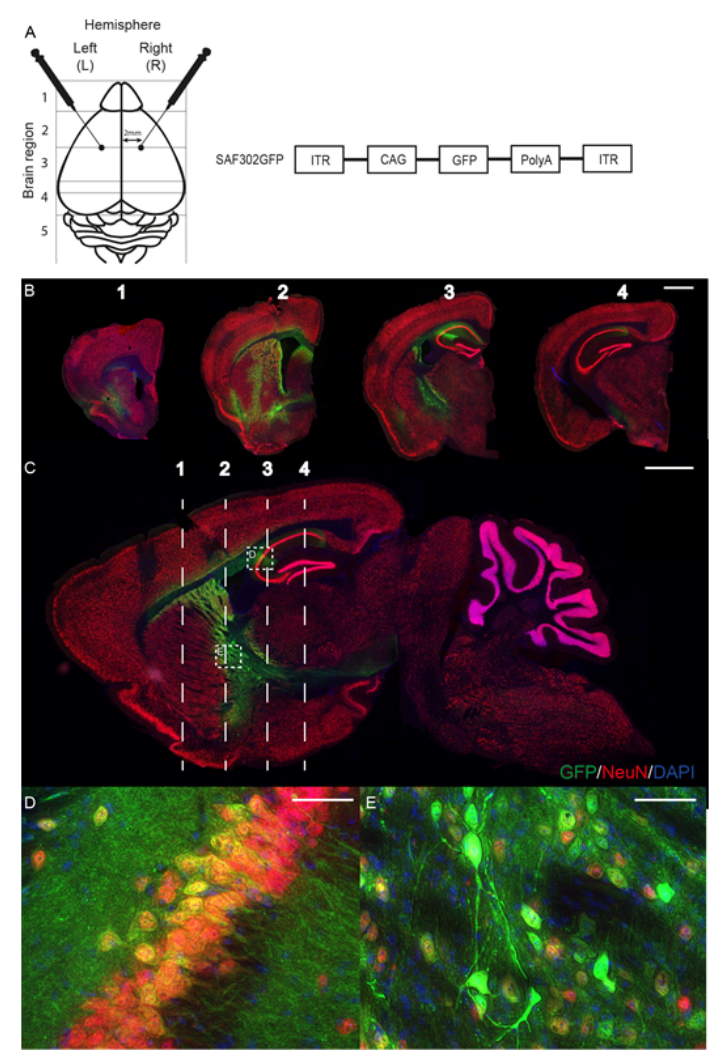

Figure 5: SAF302GFP expression is sustained at 4-months post injection in the neurons of MPSIIIA mice.

(A) MPSIIIA mice received stereotactic injection of SAF302GFP at 8-14 weeks of age using a Hamilton syringe $(n=6)$. The vector was administered at a dose of $6.1 \times 10^{9}$ genome particles in $3 \mu$ delivered via bilateral injection at a depth of $3 \mathrm{~mm}, 2 \mathrm{~mm}$ lateral to the midline in both hemispheres.

(B-C) Animals were sacrificed 4 months after injection and brains were taken for histological analyses. Coronal brain sections from Bregma $+1.7,+0.26,-1.18$ and $-2.62 \mathrm{~mm}$ (B) and a sagittal section at $1.2 \mathrm{~mm}$ lateral to the midline (C) were co-labelled with NeuN (red), GFP (green) and DAPI (blue). Scale bars $=1000 \mu \mathrm{m}$.

(D-E) High magnification images of the regions indicated by the dashed boxes in $\mathrm{C}$ show transduction of cells within the hippocampus (D) and the striatum (E). Scale bar $=50 \mu \mathrm{m}$. 

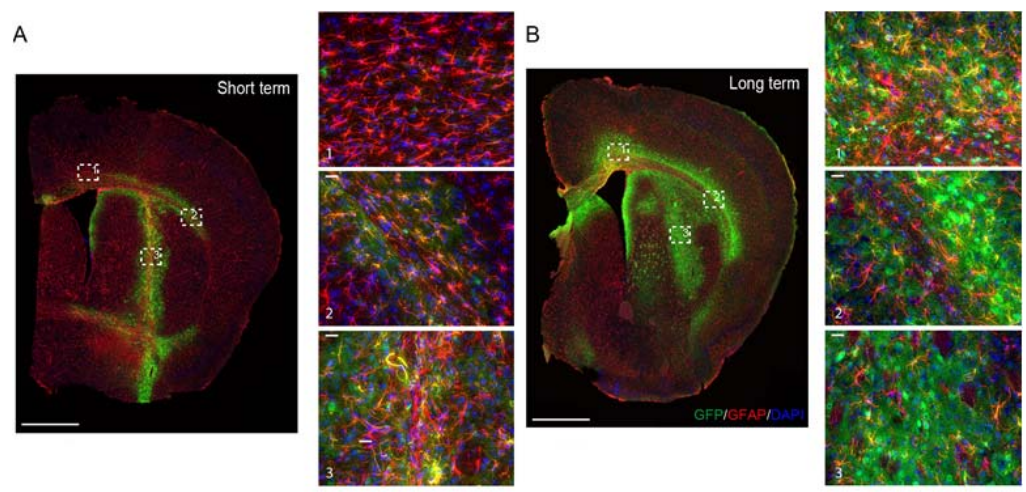

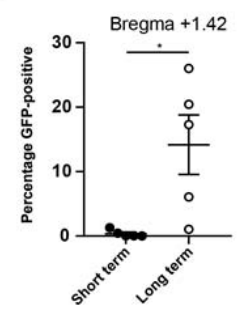

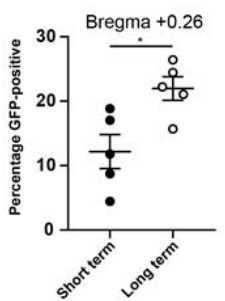

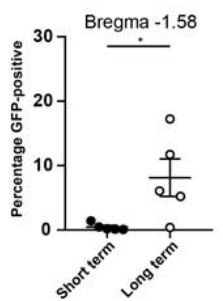

Figure 6: Clear transduction of GFAP-positive astrocytes is seen near the injection site following treatment with SAF302GFP.

(A-B) MPSIIIA mice were injected with SAF302GFP according to the protocol detailed in Figure $1 A(A$, short term mice) or Figure $5 A(B$, long-term mice) harvesting at either 4weeks (A) or 4-months (B) post injection. Coronal sections at +0.26 relative to Bregma were stained with GFAP (red), GFP (green) and DAPI (blue). Scale bars $=1000 \mu m$. High magnification images of the cingulum/cortex (1), external capsule/primary somatosensory cortex/striatum boundary (2) and striatum/injection site (3) is shown. Scale bar $=20 \mu \mathrm{m}$.

(C-E): Quantification of percentage of GFP positive cells at the indicated Bregma as a proportion of total brain area. ${ }^{*} p<0.05$ with $p$ values calculated using a student's T-test.

Representative images are shown, with 5 mice injected in both hemispheres and quantified per group. 
Supplementary Figures:

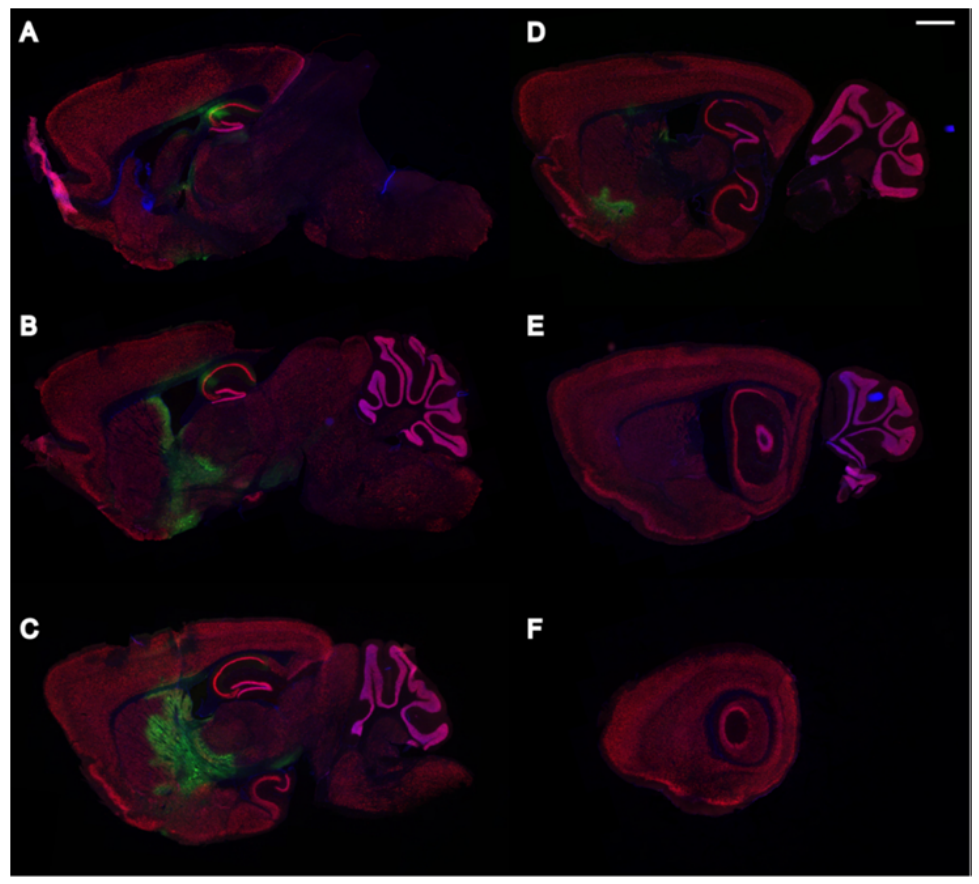

\section{Supplementary Figure 1: Distribution of SAF302GFP vector across the sagittal plane.}

(A-F) Sagittal sections throughout the hemisphere were co-stained with NeuN (red), GFP (Green) and DAPI (blue). Images were taken from positions 0.6 (A), 1.2 (B), 1.8 (C), 2.5 (D), $3(\mathrm{E})$ and $3.72(\mathrm{~F}) \mathrm{mm}$ lateral to the midline, respectively. Scale bar $=1000 \mu \mathrm{m}$. 


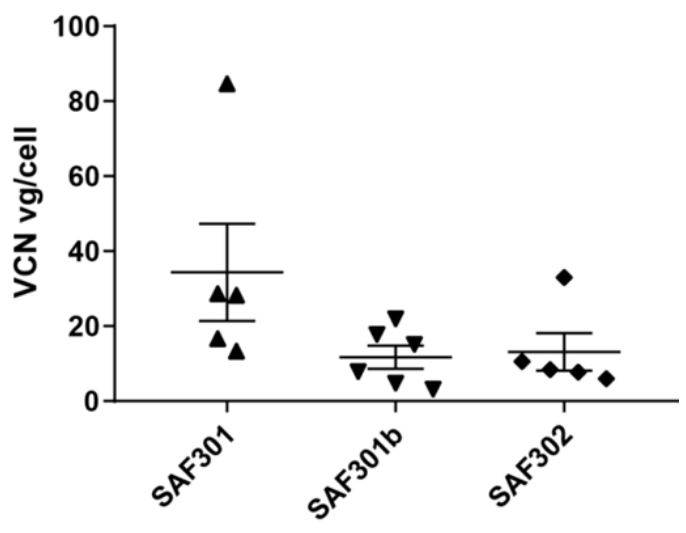

Supplementary Figure 2: No significant differences in vector copy number achieved in injected animals.

Vector copy number (VCN) in injected animals analysed by one way

ANOVA with Tukey post hoc test. Significance was set at $p<0.05$. 


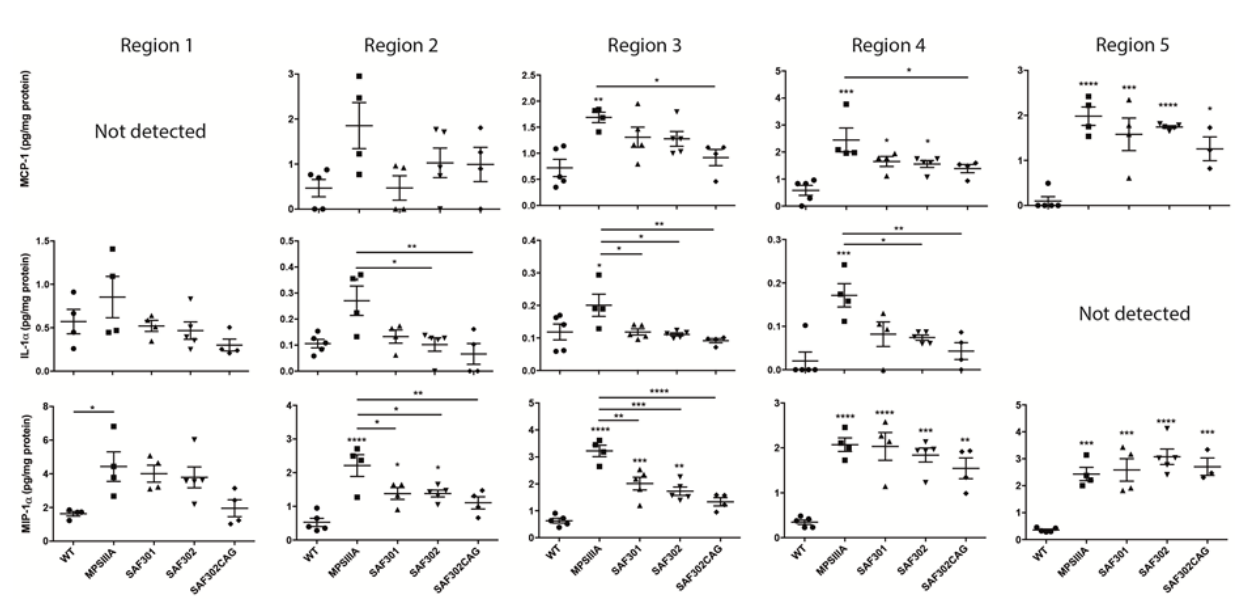

\section{Supplementary Figure 3: Assessment of neuroinflammation in brain regions 1-5.}

Brain extract from individual brain regions 1-5 were assessed for levels of inflammatory cytokines MCP-1, IL-1a and MIP-1a (A-C, respectively). Where graphs are absent, cytokine levels were not detectable in these regions. ${ }^{*} p<0.05,{ }^{* *} p<0.01, * * * p<0.001,{ }^{* * * *} p<$ 0.0001 from one way ANOVA with Tukey's multiple comparisons test. Significance to WT is shown above bars, all other comparisons are shown using a line. 